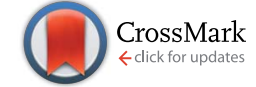

Cite this: RSC Adv., 2017, 7, 2009

Received 13th October 2016 Accepted 7th December 2016

DOI: 10.1039/c6ra25182j

www.rsc.org/advances

\section{A polycaprolactone/fish collagen/alginate biocomposite supplemented with phlorotannin for hard tissue regeneration}

\author{
JeongHyeong $\mathrm{Im},{ }^{a}$ Chang Hyun Choi, ${ }^{a}$ Frederick Mun, ${ }^{\mathrm{b}}$ JaeHwan Lee, ${ }^{\mathrm{c}}$ \\ Hyunggun Kim, ${ }^{\text {aa }}$ Won-Kyo Jung, ${ }^{\text {*d }}$ Chul Ho Jang ${ }^{\star e}$ and GeunHyung Kim ${ }^{\text {*a }}$
}

In tissue engineering, scaffolds have been widely studied for the purpose of regenerating damaged tissues or organs. Biocompatibility of materials, pore structure, and even growth factors are effective for enhancing cell growth and differentiation. In particular, mammalian collagen is biocompatible and hydrophilic, and provides good cell attachment due to interactions between Arg-Gly-Asp (RGD) domains and cell membranes. However, there have been some limitations on the use of mammalian collagen. In this study, we present a biocomposite scaffold supplemented with collagen extracted from fish skin and phlorotannin from brown algae. The effect of these biocomponents on various physical characteristics, including water wetting ability and tensile properties, was observed. The in vitro cellular activities of the biocomposites were examined using osteoblast-like cells (MG63) to assess cell proliferation and mineralization. The results show that the cells were more highly proliferated on the phlorotannin/fish collagen/alginate biocomposite compared to the control (collagen/alginate). Furthermore, the biocomposite showed significantly higher in vitro calcium deposition (38.37\%) and osteogenesis (43.2\%), determined by histomorphometric analysis, than the control. Based on these results, the biocomposite supplemented with fish collagen/phlorotannin was demonstrated to be a good potential biomaterial for enhancing bone tissue growth.

\section{Introduction}

Tissue engineering (TE) is a field in which biomedical scaffolds, cells, and growth factors are combined to regenerate or repair damaged tissues or organs. ${ }^{1}$ Scaffolds for use in regenerative applications should provide biocompatibility (to prevent adverse response), ${ }^{2}$ biodegradability (to allow the biomaterial to degrade appropriately as the tissue develops $)^{3}$ and appropriate mechanical properties (to maintain the structure throughout the regeneration process $)^{4}$ for successful regeneration of tissues or organs.

In tissue engineering applications, mammalian collagen has attracted attention due to its excellent biocompatibility and ability to promote tissue proliferation and reorganization, inducing various bioactive signals to express specific tissue-

\footnotetext{
aDepartment of Biomechatronic Engineering, College of Biotechnology and Bioengineering, Sungkyunkwan University, Suwon 440-746, Republic of Korea. E-mail: hkim.bme@skku.edu; gkimbme@skku.edu

${ }^{b}$ Department of Biological Sciences, Carnegie Mellon University, Pittsburgh, USA

${ }^{c}$ Department of Food Science and Biotechnology, Sungkyunkwan University, Suwon 440-746, Republic of Korea

${ }^{d}$ Department of Biomedical Engineering, Pukyong National University, Busan 608-737, Republic of Korea.E-mail: wkjung@pknu.ac.kr

${ }^{e}$ Department of Otolaryngology, Chonnam National University Medical School, Gwangju 500-757, Republic of Korea. E-mail: chulsavio@hanmail.net
}

related genes. ${ }^{5,6}$ However, some limitations of collagen as a biomedical material include its relatively poor physical properties (stiffness and strength), disease transmission between different species, immunogenicity, high cost, and even cultural reasons for rejecting animal-derived collagen. ${ }^{5}$ To overcome the deficiencies of collagen as a biomaterial, several researchers have tried to find new biomaterials that have biological properties similar to those of mammalian-derived collagen, ${ }^{7,8}$ and have also fabricated various biocomposites that are supplemented with synthetic biopolymers and bioceramics to increase the mechanical properties of pure collagen without losing the biological activities. ${ }^{9-11}$ Recently, as one of the typical alternatives to mammalian collagen, fish collagen (FC) has been used in tissue engineering applications. It has been reported that FC scaffolds showed outstanding cellular activity in skin regeneration ${ }^{12}$ and good osteogenesis ability. ${ }^{13}$ In addition, it is much cheaper, causes a relatively lower immune response, and is completely free from the religious limitations of mammalian collagen. ${ }^{14}$

In this study, we used collagen from different origins (porcine and fish) to prove that FC can replace porcine-derived collagen for bone tissue regeneration. To achieve this goal, we designed a functional composite scaffold, consisting of microsized poly( $\varepsilon$-caprolactone) (PCL) struts for mechanical support, phlorotannin as a bioactive component, and a collagen/alginate 
component as a release controller for the coated phlorotannin, as well as a cell-activating component. Especially the collagen/ alginate mixture was widely used due to their combination of the efficient encapsulation of growth factor and controllable delivery through rapid gelation of alginate and of the high cell inducement from the collagen component. ${ }^{15-17}$ It has been shown that in limited amounts, phlorotannin, derived from brown algae (Ecklonia cava), can promote outstanding cellular activities for regenerating bone tissues. ${ }^{18}$

To fabricate the composite scaffold, an additive manufacturing (AM) technique, which is one of the most attractive fabrication methods to manufacture complex 3D structures, and simple coating method were used. Throughout the fabrication processes, four different PCL-based composite scaffolds were obtained: (a) as a control, PCL mesh-structure coated with collagen/alginate (C/A), (b) PCL mesh-structure coated with phlorotannin (P), (c) PCL mesh-structure coated with phlorotannin and then recoated with collagen/alginate $(\mathrm{P} /$ C/A) and (d) PCL mesh structure coated with phlorotannin and then recoated with fish collagen/alginate (P/FC/A). Osteoblastlike cells (MG63) were seeded on the composite scaffolds for in vitro cellular assessment, including live/dead, DAPI/ phalloidin, and alizarin red S (ARS) to observe cell proliferation and differentiation. Finally, the scaffolds were used for in vivo study of ectopic bone formation.

\section{Experimental}

\subsection{Materials}

PCL $\left(M_{\mathrm{w}}=45000\right)$ was purchased from Sigma-Aldrich (St. Louis, MO, USA). Collagen solution (type I collagen, MatrixenPSP; Bioland Ltd., South Korea) and fish collagen were prepared at a fixed concentration of $2 \mathrm{wt} \%$. Low-viscosity, highG-content nonmedical-grade LF10/60 alginate (FMC BioPolymer, Drammen, Norway) was also used at $2 \mathrm{wt} \%$, and a collagen : alginate mixture $(1: 1)$ was used to coat the scaffold. Fish collagen was extracted from a flatfish (Paralichthys olivaceus) collected from South Korean seas, and supplied by Prof. W-K Jung (Pukyong National University, South Korea). Detailed isolation and characterization methods have been fully described previously. ${ }^{19}$ To cross-link the collagen component, it was immersed in a $50 \mathrm{mM}$ 1-ethyl-(3-3-dimethylaminopropyl) carbodiimide hydrochloride (EDC, $M_{\mathrm{w}}=191.7$; Sigma-Aldrich) solution in $95 \%$ ethanol for $4 \mathrm{~h}$ at room temperature, and 5 wt $\% \mathrm{CaCl}_{2}$ (Sigma-Aldrich) was used to cross-link the alginate.

The brown seaweed E. cava was collected along the Jeju Island coast of Korea from October 2008 to March 2009. Fresh $E$. cava was washed three times with tap water to remove salt, epiphytes, and sand attached to the surface of the samples and stored at $-20{ }^{\circ} \mathrm{C}$. The frozen samples were lyophilized and homogenized using a grinder before extraction. Phlorotannins were isolated from $E$. cava powder as described previously by Heo et al., ${ }^{20}$ with slight modifications. Briefly, the dried EC powder (500 g) was extracted three times with $80 \% \mathrm{MeOH}$ and then filtered. The filtrate was evaporated at $40{ }^{\circ} \mathrm{C}$ to obtain the methanol extract. Next, the extract was suspended in distilled water and partitioned with ethyl acetate. The ethyl acetate fraction was mixed with celite, dried and packed into a glass column, and eluted in the order of hexane, methylene chloride, diethyl ether, and methanol. The diethyl ether fraction was further purified by Sephadex LH-20 column chromatography using a stepwise gradient chloroform/methanol $(2 / 1 \rightarrow 0 / 1)$ solvent system. The extracted phlorotannin was dissolved in ethanol at $5 \mathrm{wt} \%$. In our previous work, the $5 \mathrm{wt} \%$ phlorotannin significantly influenced in vitro bone formation. ${ }^{21}$

\subsection{Fabrication of composite scaffolds}

A three-axis robot system supplemented with a melting dispenser can manufacture multi-layered porous PCL structures with constant pore and strut diameters. The pore size, $350 \mu \mathrm{m}$, was set for osteoconduction and bone formation through vascularization. As shown in Fig. 1, perpendicular-layered PCL scaffolds $(28 \times 28 \times 1.2 \mathrm{~mm})$ were fabricated using the meltprinting method (temperature $=100{ }^{\circ} \mathrm{C}$, pressure $=500 \mathrm{kPa}$, nozzle speed $=0.4 \mathrm{~mm} \mathrm{~s}^{-1}$ ). After fabrication, the coating process was performed by dipping the PCL scaffold in phlorotannin solution and drying for $24 \mathrm{~h}$. The coated sample was washed five times with distilled water. After drying the scaffold, the weight fraction of phlorotannin in $\mathrm{EtOH}$ was measured as 5 $\mathrm{wt} \%$ for the coated phlorotannin solution. After coating the phlorotannin on the scaffold, it was recoated with either collagen/alginate (C/A) or fish collagen/alginate solution (FC/A).

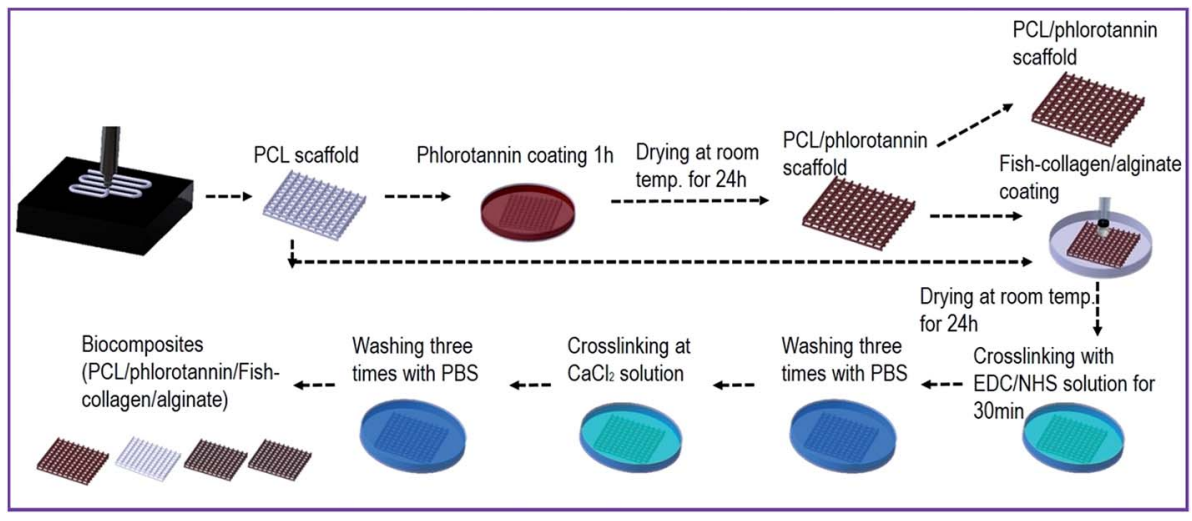

Fig. 1 Schematic representation of the fabrication processes for producing biocomposites containing phlorotannin/fish collagen/alginate. 


\subsection{Characterizations of the composite scaffolds}

The morphology of the scaffolds was visualized by scanning electron microscopy (SEM) (Sirion; FEI, USA). The pore size was defined as the distance between the struts and analyzed using the SEM images.

The water uptake ability was calculated by weighing the scaffolds before and after soaking in distilled water for $12 \mathrm{~h}$. The percentage increase in water absorption was calculated as $(\%)=$ $\left(W_{12 \mathrm{~h}}-W_{\mathrm{o}}\right) / A \times 100$, where $W_{12 \mathrm{~h}}$ is the weight of scaffolds after $12 \mathrm{~h}, W_{\mathrm{o}}$ is the weight at time zero, and $A$ is the area of scaffold.

To observe the biodegradability of the coated component, the initial weight $\left(W_{\mathrm{i}}\right)$ of the scaffolds that were only coated with the collagen/alginate or fish-collagen/alginate was measured, and then those were immersed in PBS ( $\mathrm{pH} \mathrm{7.4)} \mathrm{at} 37^{\circ} \mathrm{C}$. After incubation for 7 days, the scaffolds were freeze-dried and their weight $\left(W_{\mathrm{f}}\right)$ was measured. Because the PCL cannot be degraded for 7 days, we assume the most weight loss was from the collagen/ alginate component. The percent weight loss was calculated with the following equation, weight loss $(\%)=\left[\left(W_{\mathrm{i}}-W_{\mathrm{f}}\right) / W_{\mathrm{i}}\right] \times$ 100. Weight loss is shown as the mean \pm SD of five samples.

Phlorotannin released from the composites was monitored by UV-spectrophotometry (Optizen 2120UVPlus, Mecasys Co., Daejeon, South Korea) in PBS (pH 7.4) at a wavelength of $301 \mathrm{~nm}$. The phlorotannin release test was carried out by incubating the scaffold in $15 \mathrm{~mL}$ of PBS solution at $37^{\circ} \mathrm{C}$ for various time periods. To measure phlorotannin release, $1 \mathrm{~mL}$ of the solution was removed and tested; the aliquot was then returned to the release solution. To avoid contamination of release solution, the pipette tip and cuvette of the spectrophotometer were sterilized with $70 \%$ ethanol. The cumulative amount of phlorotannin released was calculated as a function of time.

To assess the mechanical properties, the scaffolds were cut into small strips $\left(5 \times 15 \mathrm{~mm}^{2}\right)$. For each $3 \mathrm{D}$ scaffold, five samples were taken from different sites. A tensile test was carried out using a tensile machine (Top-tech 2000; Chemilab, South Korea). The stress-strain curves for the scaffolds were recorded at a stretching speed of $0.5 \mathrm{~mm} \mathrm{~s}^{-1}$. All values are mean $\pm \mathrm{SD}(n=5)$.

The fabricated composite scaffolds were immersed in $1 \times$ simulated body fluid (SBF), consisting of $\mathrm{NaCl}$ (7.995 g), $\mathrm{KCl}$ (0.224 g), $\mathrm{CaCl}_{2} \cdot 2 \mathrm{H}_{2} \mathrm{O}$ (0.368 g), $\mathrm{MgCl}_{2} \cdot 6 \mathrm{H}_{2} \mathrm{O}$ (0.305 g), $\mathrm{K}_{2} \mathrm{HPO}_{4}$ (0.174 g), $\mathrm{NaHCO}_{3}(0.349 \mathrm{~g})$, and $\mathrm{Na}_{2} \mathrm{SO}_{4} \cdot 10 \mathrm{H}_{2} \mathrm{O}(0.161 \mathrm{~g})$ in $1 \mathrm{~L}$ of distilled water. The $\mathrm{pH}$ was adjusted to 7.4 by addition of Tris-HCl. The composite scaffolds were added to the fluid and remained there during incubation at $37{ }^{\circ} \mathrm{C}$. After 7 days, the scaffolds were washed with deionized water to remove adsorbed minerals, and lyophilized.

To measure the crystallite size of the biomineralized surface of the fabricated scaffolds, wide-angle X-ray diffraction (WAXD) was performed using a Siemens D 500 WAXD (Siemens, Munich, Germany) with $\mathrm{CuK}_{\alpha}$ radiation under beam conditions of $40 \mathrm{kV}$ and $20 \mathrm{~mA}$ with spectra collection at $2 \theta=5-80^{\circ}$ and a step size of $0.1^{\circ}$.

\subsection{In vitro cell culture}

Fabricated scaffolds $\left(5 \times 5 \mathrm{~mm}^{2}\right)$ were sterilized with $70 \%$ ethanol and ultraviolet (UV) light. MG63 cells (MG63-human source, ATCC number CRL-1427; ATCC, Manassas, VA, USA) were used to evaluate the behavior of cells cultured in the scaffolds. The cells were cultured for up to nine passages in 24well plates containing Dulbecco's modified Eagle's medium (DMEM; Hyclone, USA) supplemented with 10\% fetal bovine serum (Hyclone) and 1\% penicillin-streptomycin (Hyclone). The cells were collected by trypsin-ethylenediaminetetraacetic acid (EDTA) treatment, seeded onto the scaffolds at a density of $1 \times 10^{5}$ per specimen, and incubated at $37{ }^{\circ} \mathrm{C}$ in an atmosphere of $5 \% \mathrm{CO}_{2}$. The medium was changed every second day.

\subsection{Live/dead cell staining}

After 1, 3 and 7 days of cell culture, the scaffolds were exposed to $0.15 \mathrm{mM}$ calcein AM and $2 \mathrm{mM}$ ethidium homodimer- 1 for $1 \mathrm{~h}$ in an incubator to permit observation of live and dead cells. The stained specimens were visualized under a microscope (TE2000S; Nikon, Tokyo, Japan) with an epifluorescence attachment and a SPOT RT digital camera (SPOT Imaging Solutions, Sterling Heights, MI). Stained images were captured in which green and red indicated live and dead cells, respectively. Image J software (National Institutes of Health, Bethesda, MD) was utilized to enumerate live cells in the width direction of the scaffolds after 7 days of culture.

\subsection{DAPI/phalloidin staining}

After 7 and 14 days of cell culture, the scaffolds were analyzed with diamidino-2-phenylindole (DAPI; dilution ratio of $1: 100$, Invitrogen, Carlsbad, CA, USA) staining to characterize the nuclei of the cells. Alexa Fluor 568 Phalloidin (dilution ratio of $1: 100$, Invitrogen) was used to visualize the actin cytoskeleton of the cells in the scaffolds by fluorescence microscopy (CKX41; Olympus).

\subsection{Alizarin red S staining}

Calcium mineralization was determined by alizarin red $\mathrm{S}$ staining of MG63 cells cultured on the scaffolds in 24-well plates. The cells were washed three times with PBS, fixed in $70 \%$ (v/v) cold ethanol $\left(4^{\circ} \mathrm{C}\right)$ for $1 \mathrm{~h}$, and air-dried. The ethanol-fixed specimens were stained with $40 \mathrm{mM}$ alizarin red $\mathrm{S}(\mathrm{pH} 4.2)$ for $1 \mathrm{~h}$ and washed three times with purified water. Specimens were then destained with $10 \%$ cetylpyridinium chloride in $10 \mathrm{mM}$ sodium phosphate buffer $(\mathrm{pH} 7.0)$ for $30 \mathrm{~min}$. An optical microscope was used to observe the extent of staining, and the OD was measured at $562 \mathrm{~nm}$ using a Spectra III UV microplate reader. The OD was normalized to the total protein content (OD value).

\subsection{In vivo study for ectopic bone formation}

All animal experiments followed a protocol approved by the Committee for Animal Experimentation at Chonnam National University, Korea (CNU IACUC-H-2016-131). We divided fourteen normal $(\mathrm{BALB} / \mathrm{C})$ mice into two groups according to the type of scaffold: control group $(n=7, \mathrm{C} / \mathrm{A})$ and study group $(n=$ 7, P/FC/A). General anesthesia was administered by intraperitoneal injection of a mixture of Zoletil $\left(30 \mathrm{mg} \mathrm{kg}^{-1}\right.$; Virbac Lab, 
Carros, France) and Rompun (10 mg kg ${ }^{-1}$; Bayer Korea Ltd, Ansan, Korea). Each mouse was shaved at the thigh region and painted with povidone-iodine and ethanol.

Following unilateral posteromedial skin incisions along the right thigh, a muscle pocket was created in each hind limb. The scaffold was inserted into the muscle pocket. After suturing, intramuscular injection of ciprofloxacin was administered.

We harvested thigh muscles containing ectopic bone at 8 weeks after intramuscular implantation to evaluate the histology of the tissues. The round-shaped ectopic bone was isolated after dissection. We carefully detached the muscles and soft tissue affixed to each round ectopic bone mass.

The ectopic bone samples were fixed in $10 \%$ formalin solution, decalcified by graded dehydration, and then embedded in paraffin and cut into serial sections with a thickness of $5 \mathrm{~mm}$. The sections were stained with hematoxylin and eosin for examination under a light microscope. Histomorphometric analysis was performed using an image analysis system (Image Inside, Focus Technology, Daejon, Korea).

\subsection{Statistical analysis}

All data presented are expressed as mean \pm SD. Statistical analyses comprised single-factor analyses of variance (ANOVA). The significance level was set at $p<0.05$. For in vivo work, the one-way ANOVA test was employed for statistical analysis. The statistical significance was set at $5 \%$ and $p$-values were adjusted for multiple comparisons.

\section{Results and discussion}

\subsection{Micro-/internal structure of scaffold}

The optical and SEM images of the fabricated scaffolds are shown in Fig. 2(a-d) and $3(\mathrm{a}-\mathrm{d})$, respectively. In the optical images the yellowish color of the scaffolds (P, P/C/A and P/FC/A) was clearly shown to emphasize the coated phlorotannin component. However, pores of the scaffold (P/C/A) are partly blocked due to the non-homogeneous coating-ability, which is because the porcine collagen could be reacted with tannin component in the phlorotannin.

It has been shown that the pore size and surface chemistry of biomedical scaffolds directly influence cell adhesion and proliferation. ${ }^{22,23}$ Generally, it is well known that the appropriate pore size of biomedical scaffolds to induce osteogenic differentiation is between 300 and $500 \mu \mathrm{m} .{ }^{20}$ Based on this, we set the pore and strut size of the manufactured scaffolds as $350 \mu \mathrm{m}$ and $250 \mu \mathrm{m}$ and, as shown in the surface and cross-sectional optical images, the designed pore structure was well fabricated. We used the SEM images to precisely measure the pore size and observe the surface morphology, and the pore and strut size were found to be about $360 \pm 18 \mu \mathrm{m}$ and $265 \pm 21 \mu \mathrm{m}$, respectively, confirming that the pore structure was wellfabricated. The surface of the struts was also highly roughened due to the coating components. As expected, the $\mathrm{C} / \mathrm{A}, \mathrm{P} / \mathrm{C} /$ A and P/FC/A scaffolds were slightly thicker, due to the mixture of collagen/alginate, than the P scaffold. Using the SEM images, we quantitatively measured the average coated layers of C/A (2 $\mu \mathrm{m}), \mathrm{P}(0.5 \mu \mathrm{m}), \mathrm{P} / \mathrm{C} / \mathrm{A}(2 \mu \mathrm{m})$ and $\mathrm{P} / \mathrm{FC} / \mathrm{A}(2 \mu \mathrm{m}$ thick $)$ on the scaffold surfaces.

\subsection{Scaffold characteristics}

To observe the in vitro biomineralization ability of the scaffolds, they were immersed in $1 \times$ SBF for 7 days. Fig. 4(a and b) show SEM and energy-dispersive spectroscopy (EDS) images taken before and after in vitro biomineralization of the $\mathrm{C} / \mathrm{A}$ and $\mathrm{P} / \mathrm{FC} / \mathrm{A}$ scaffolds. In the EDS mapping images, the green and red dots indicate "Ca" and " $\mathrm{P}$ ", respectively, and the " $\mathrm{Ca} / \mathrm{P}$ " ratio on the scaffolds (C/A and P/FC/A) was about 1.65 and 1.53, respectively, which are similar value of hydroxyapatite. As shown in the both SEM images of C/A and P/FC/A scaffold, the mineralized apatite particles are shown. However, the coated region of the collagen/ alginate on the surface of the C/A scaffold was not completely homogenous, but the $\mathrm{P} / \mathrm{FC} / \mathrm{A}$ scaffold showed a relatively (a) $\mathrm{C} / \mathrm{A}$

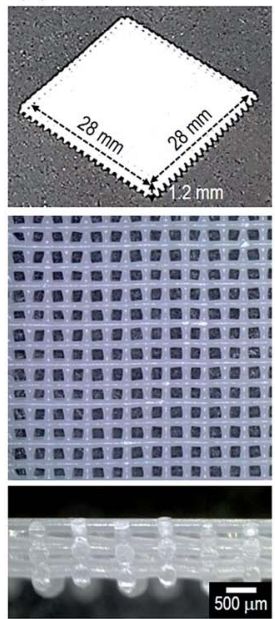

(b) $P$

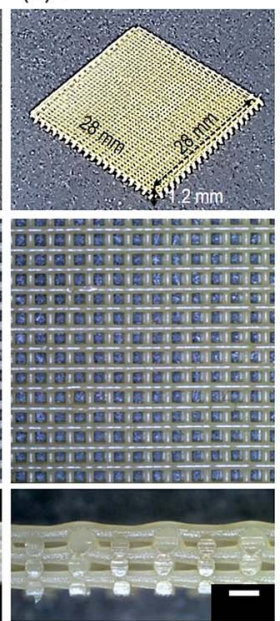

(c) $P / C / A$

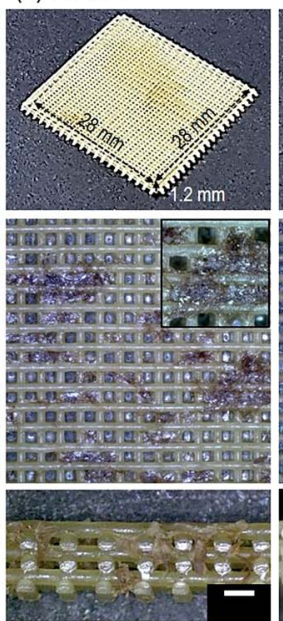

(d) P/FC/A

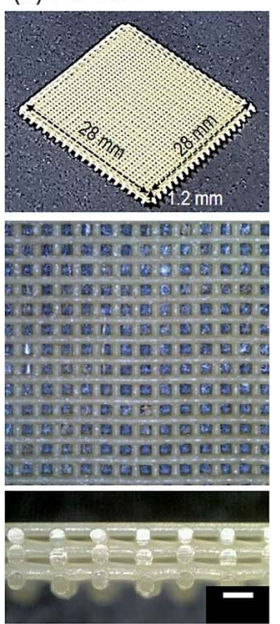

Fig. 2 Optical microscope images of PCL-based biocomposites coated with (a) collagen/alginate (C/A), (b) phlorotannin, (c) phlorotannin/ collagen/alginate (P/C/A) and (d) phlorotannin/fish collagen/alginate (P/FC/A). 
(a) $\mathrm{C} / \mathrm{A}$

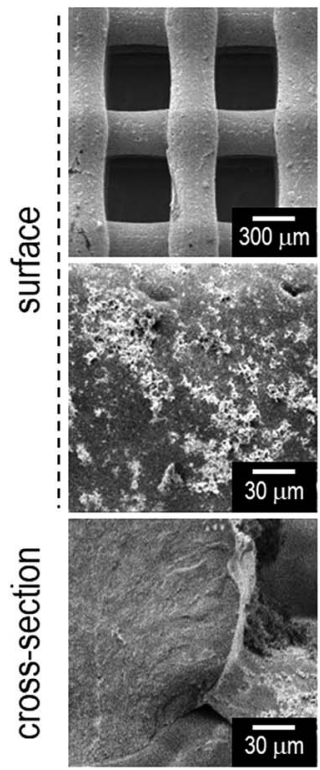

(b) $P$
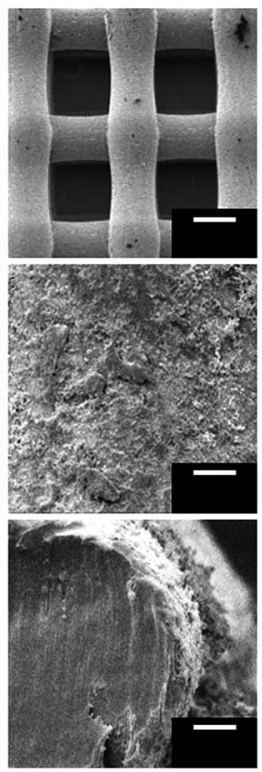

(c) $P / C / A$

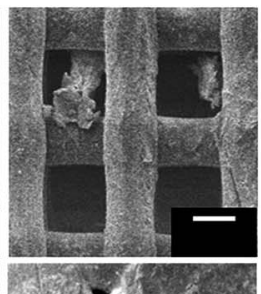

(d) P/FC/A

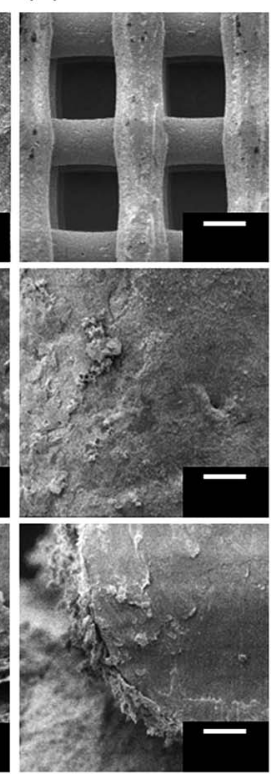

Fig. 3 SEM images of (a) collagen/alginate (C/A), (b) phlorotannin (P), (c) phlorotannin/collagen/alginate (P/C/A) and (d) phlorotannin/fish collagen/alginate (P/FC/A) biocomposites.

(a)

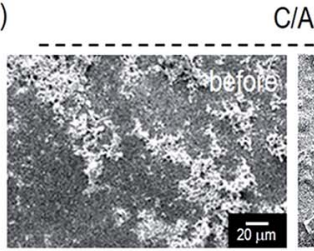

C/A

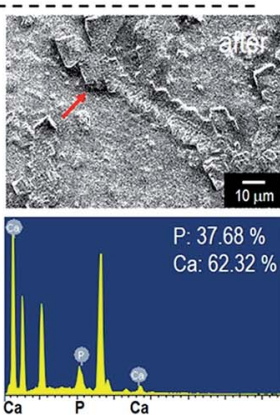

(b)

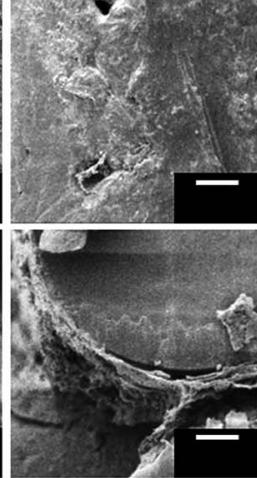

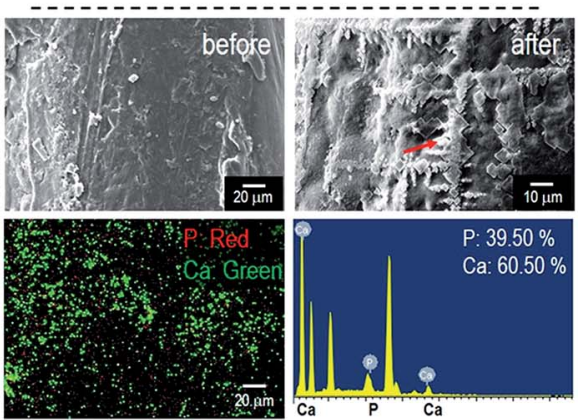

(c)

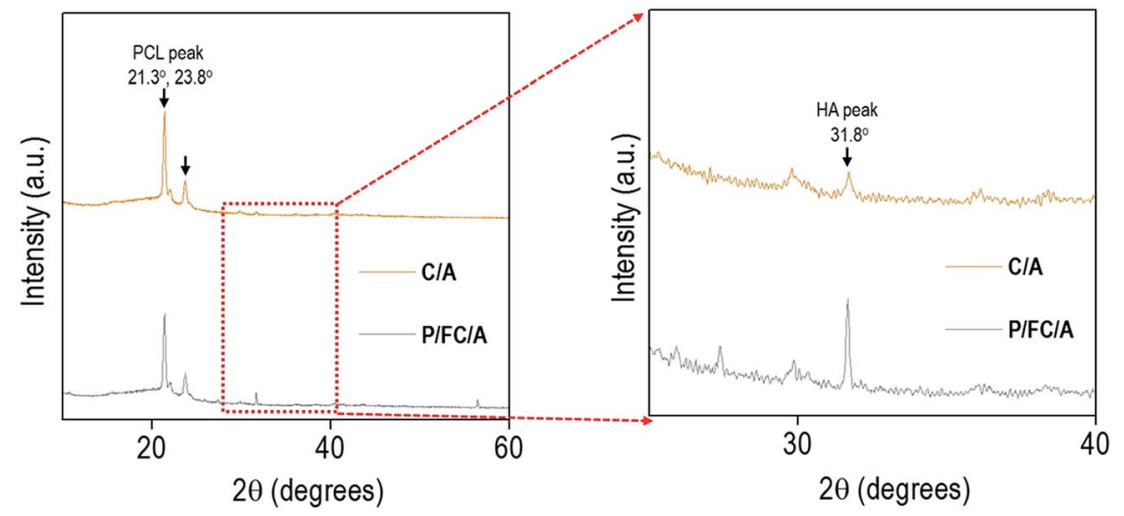

Fig. 4 Surface SEM, Ca/P mapping images, and EDS spectra taken before and after in vitro mineralization of (a) collagen/alginate (C/A) and (b) phlorotannin/fish collagen/alginate (P/FC/A) scaffolds. Red arrows indicate the hydroxyapatite particles. (c) X-ray diffraction (XRD) patterns for C/ $A$ and $P / F C / A$.

homogeneously coated surface. It is known that surface roughness homogeneity can directly influence in vitro biomineralization ability, even for surfaces with similar chemical compositions. ${ }^{24}$ As shown in the SEM image of the C/A scaffold, developed apatite particles were obtained, but the covering was deficient over the whole strut surface (Fig. 4(a)). However, for 
the P/FC/A scaffold, the apatite particles on the surface were more evenly developed (Fig. 4(b)). This phenomenon could be due to the different coating ability of the coating agents; the fish collagen has a significantly higher solubility in dilute acid and mammalian collagen has lower solubility. ${ }^{\mathbf{1 4 2 5}}$

To quantitatively measure the apatite crystallite size on the strut surface, X-ray diffractometer analysis of the strut surfaces was performed and the results are shown in Fig. 4(c). It is well known that the high diffraction peaks $\left(21.3^{\circ}\right.$ and $\left.23.8^{\circ}\right)$ are due to the orthorhombic planes, (110) and (200), of the PCL struts, while the peak $\left(31.8^{\circ}\right)$ was attributed to the crystallite plane, (211), which is generated from the developed hydroxyapatite (HA). ${ }^{26}$ As shown in the magnified graphs, the peak intensity at $31.8^{\circ}$ of the $\mathrm{P} / \mathrm{FC} / \mathrm{A}$ scaffold was significantly higher than that of the scaffold (C/A). In addition, the full-width at half-maximum (FWHM) was measured using the diffracted plane (211) and crystallite size was measured using the Scherer equation, $0.89 \lambda /$ $[\beta \cos (\theta)]$. In the equation, ' $\lambda$ ' is wavelength $(0.154 \mathrm{~nm})$, ' $\beta$ ' is FWHM, and ' $\theta$ ' is the diffraction angle. The mean size of the crystallites was significantly higher in the P/FC/A scaffold (127 $\mathrm{nm})$ relative to the $\mathrm{C} / \mathrm{A}$ scaffold $(103 \mathrm{~nm})$. The larger crystallite size of the $\mathrm{P} / \mathrm{FC} / \mathrm{A}$ could be due not only to the homogeneously coated FC/A mixture, but also the synergistic effect of the coated phlorotannin component attracting $\mathrm{Ca}^{2+}$, indicating that the scaffold can induce significant osteogenesis to accelerate hard tissue regeneration.

The hydrophilic properties of a scaffold play an important role in promoting cellular activities including initial cell attachment and proliferation. ${ }^{27-29}$ Therefore, numerous studies have modified the surface chemical composition by coating with biopolymers such as collagen ${ }^{25}$ and alginate. ${ }^{26}$ The optical images in Fig. 5(a) show the water wetting of the scaffolds to determine their hydrophilicity. All scaffolds showed outstanding wetting ability due to the hydrophilic coating components (phlorotannin and collagen/alginate). Moreover, the water absorption ability of the scaffolds showed no significant difference (Fig. 5(b)). Based on these results, we confirmed that both the control $(\mathrm{C} / \mathrm{A})$ and experimental groups $(\mathrm{P}, \mathrm{P} / \mathrm{C} / \mathrm{A}$ and $\mathrm{P} / \mathrm{FC} / \mathrm{A}$ ) had sufficient hydrophilic properties to promote initial cellular activities including efficient cell seeding and cell adhesion/spreading.

The mechanical stiffness of biomedical substitutes should be analogous to those of the environmental tissues to avoid stress-concentration in the affected region, and stiffness can also directly affect cell growth and differentiation. ${ }^{30}$ Collagen has been used extensively for soft/hard tissue regeneration since it is the main organic component of bone and one of the main components of the extracellular matrix (ECM). ${ }^{31}$ Although collagen can be considered an ideal component for tissue regeneration, the main weakness of the material is its low mechanical property, so it has been applied in composites and blending systems using biocompatible ceramics and synthetic polymers. ${ }^{9-11}$ To improve the shortcomings of the collagen/ alginate, we used PCL as a structural support. Fig. 5(c) shows the tensile stress-strain curves of the scaffolds with a stretching speed of $0.5 \mathrm{~mm} \mathrm{~s}^{-1}$. The scaffolds showed a completely similar mechanical behavior, with linear elastic regions and then fluctuating regions due to strain hardening of each strut. As expected, the elastic moduli of the biocomposites were not significantly different $(p<0.05)$ (Fig. 5(d)).

To observe the biodegradability of the collagen/alginate and fish-collagen/alginate mixtures, weight loss of the scaffolds that were only coated with collagen/alginate (C/A) and fish-collagen/ alginate (FC/A) was measured after 7 days in the PBS solution at $37{ }^{\circ} \mathrm{C}$. The degradation of the $\mathrm{C} / \mathrm{A}(5.6 \pm 1.2 \%)$ and $\mathrm{FC} / \mathrm{A}(6.1 \pm$ $1.6 \%)$ scaffolds was similar.
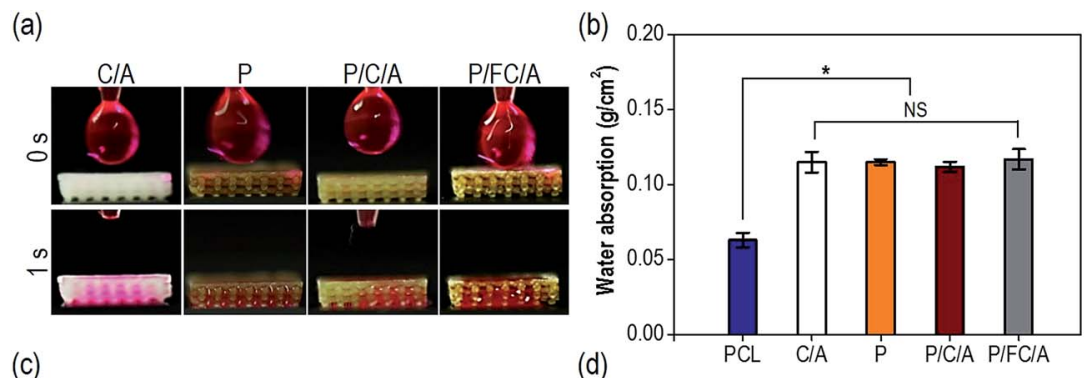

(c)

(d)
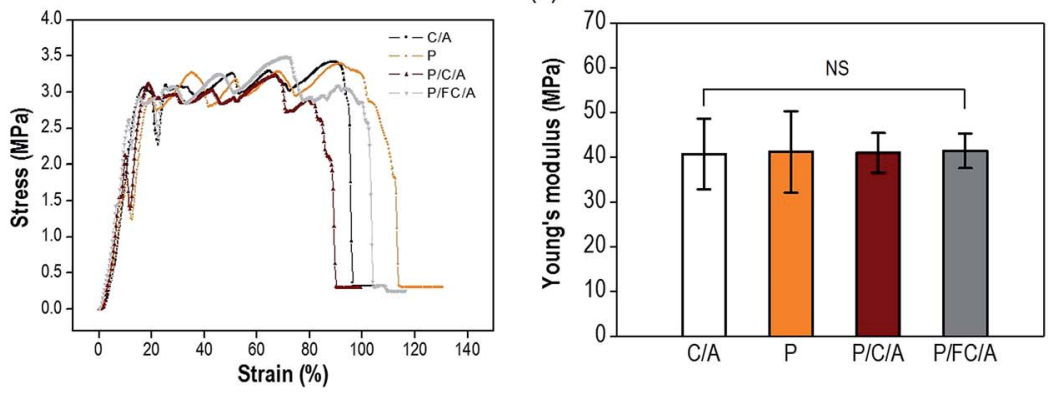

Fig. 5 (a) Transient absorption images of one droplet ( $5 \mu \mathrm{L}$ ) of water through the cross-section of the scaffolds. (b) Graph comparing water absorption for the various biocomposites $(n=5)$. (c) Stress-strain curves for the biocomposites and (d) measured moduli. PCL, poly $(\varepsilon-c a p r o-$ lactone); C/A, collagen/alginate; P, phlorotannin; P/C/A, phlorotannin/collagen/alginate; P/FC/A, phlorotannin/fish collagen/alginate. * indicates $p<0.05$ and NS indicates nonsignificance. 

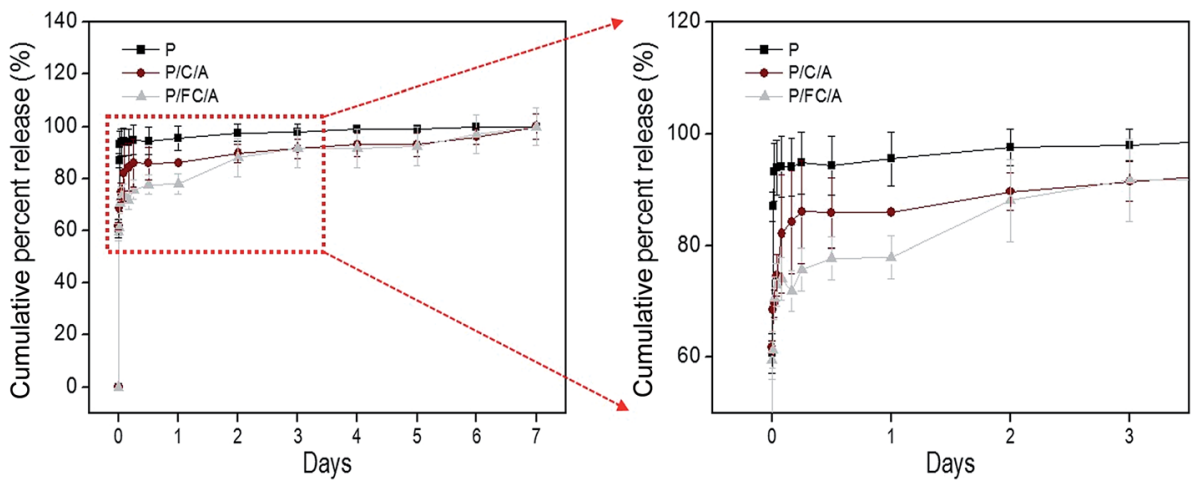

Fig. 6 Cumulative percent release of phlorotannin from the phlorotannin (P), phlorotannin/collagen/alginate $(\mathrm{P} / \mathrm{C} / \mathrm{A})$ and phlorotannin/fish collagen/alginate (P/FC/A) biocomposites.

Recently, scaffolds combined with drug delivery systems (DDS) have been widely applied in tissue regeneration. ${ }^{32-34}$ To effectively regulate the release of biocompatible components, controllable release is required. In our previous works, we investigated the controlled release of various bioactive components, such as fucoidan ${ }^{35}$ and sulfuretin, ${ }^{36}$ using a coated alginate to avoid an initial biocomponent burst effect. In this work, we also employed a similar system using the coated collagen/ alginate component. Fig. 6 displays the cumulative percent release of phlorotannin from the $\mathrm{P}, \mathrm{P} / \mathrm{C} / \mathrm{A}$ and $\mathrm{P} / \mathrm{FC} / \mathrm{A}$ scaffolds. Most of the phlorotannin in the $\mathrm{P}$ scaffold without the coating component was released until $1 \mathrm{~h}$. However, phlorotannin release from the P/FC/A scaffold was delayed with the coated component (fish collagen/alginate) and lasted 6-7 days, although $80 \%$ of the bioactive component was released in the first $12 \mathrm{~h}$. Based on this result, we can confirm that the fish collagen/alginate layer can delay the phlorotannin release. In addition, as the comparing the release behavior of the $\mathrm{P} / \mathrm{C} / \mathrm{A}$ and $\mathrm{P} / \mathrm{FC} / \mathrm{A}$, the phlorotannin release of the $\mathrm{P} / \mathrm{FC} / \mathrm{A}$ was meaningfully delayed relative to that of $\mathrm{P} / \mathrm{C} / \mathrm{A}$. This phenomenon was because of the homogenous coat-ability of the fish collagen/ alginate, which was shown in the Fig. 2(d), compared to that of the P/C/A of Fig. 2(c). We can estimate that the bioactive component (phlorotannin) in the $\mathrm{P} / \mathrm{FC} / \mathrm{A}$ scaffold can be continuously released for 7 days. To measure the released amount of phlorotannin at 7 days, we used a standard curve (the optical densities vs. the various concentrations of phlorotannin). By using the curve, we can obtain the total released amount of phlorotannin from the scaffolds (size: $5 \times 5 \times 1.2$ $\left.\mathrm{mm}^{3}\right)$ at 7 days, $2.52 \mathrm{mg}(\mathrm{P}), 0.59 \mathrm{mg}(\mathrm{P} / \mathrm{C} / \mathrm{A})$, and $0.53 \mathrm{mg}(\mathrm{P} / \mathrm{FC} /$ A). The small amount of the coated phlorotannin of the $\mathrm{P} / \mathrm{C} / \mathrm{A}$ and $\mathrm{P} / \mathrm{FC} / \mathrm{A}$ scaffolds was because the more complex crosslinking/washing process was applied during the scaffold fabrication.

\subsection{In vitro tests}

To observe in vitro cellular responses to the scaffolds, osteoblast-like cells (MG63) were cultured on them. Cell proliferation is an important factor when evaluating scaffolds because it directly influences successful tissue regeneration. Fig. 7 (a) shows the total protein content, indicating proliferated cells, after 7 and 14 days culture periods. To remove the effect of the collagen content of the scaffold, the initial collagen content was measured and subtracted. A significant increase was observed with the $\mathrm{P} / \mathrm{FC} / \mathrm{A}$ and $\mathrm{P} / \mathrm{C} / \mathrm{A}$ scaffold compared to the control, C/A scaffold.

To measure live/dead cells on the scaffolds, they were stained with calcein AM (live) and ethidium homodimer-1 (dead). Fig. 7(b) shows fluorescence images of live (green) and dead (red) cells on the scaffolds after 7 days. Similar to the protein content results, the proliferated live cells were more distributed on the surface of the $\mathrm{P} / \mathrm{C} / \mathrm{A}$ and $\mathrm{P} / \mathrm{FC} / \mathrm{A}$ scaffold compared to the other scaffolds ( $\mathrm{P}$ and $\mathrm{C} / \mathrm{A})$. Using the live/dead images taken after 1, 3, and 7 days, we measured the cell-density for each scaffold (Fig. 7(c)). As expected, the cell-density for the same area was significantly higher in the $\mathrm{P} / \mathrm{FC} / \mathrm{A}$ scaffold. We think this result was due to the synergistic effect of continuously released phlorotannin and the $\mathrm{FC} / \mathrm{A}$ component facilitating the proliferation of the osteoblast-like cells.

The images in Fig. 7(d) show the nuclei (blue) and F-actin (red) of the proliferated cells on the scaffolds, which were obtained after 7 and 14 days of cell culture. Increased numbers of nuclei and a more developed cytoskeleton were clearly observed in the $\mathrm{P} / \mathrm{FC} / \mathrm{A}$ scaffold, while the control, $\mathrm{P}$, and $\mathrm{P} / \mathrm{C} / \mathrm{A}$ scaffolds showed relatively low activities (Fig. $7(\mathrm{e}$ and $\mathrm{f})$ ). This result shows that the $\mathrm{P} / \mathrm{FC} / \mathrm{A}$ scaffold combined with phlorotannin can induce meaningful cellular activities, including cytoskeleton development. Also, as comparing the results of $\mathrm{P} / \mathrm{C} / \mathrm{A}$ and $\mathrm{P} / \mathrm{FC} /$ A scaffolds, the cytoskeleton activities were more significant in the $\mathrm{P} / \mathrm{FC} / \mathrm{A}$ than those of $\mathrm{P} / \mathrm{C} / \mathrm{A}$ scaffold. We think the relatively low activities of the $\mathrm{P} / \mathrm{C} / \mathrm{A}$ scaffold was occurred due to the nonhomogenous coating of collagen/alginate, which was because the porcine collagen was reacted with the phlorotannin. ${ }^{37}$

In general, calcium deposition results have been used to evaluate the level of calcium mineral in cell culture scaffolds. In Fig. 8(a), optical images of ARS staining of the scaffolds after cell culture for 7 and 14 days are shown. In the images, the red color defines the area of calcium mineral deposition. With increasing culture time, a denser color was observed in all scaffolds. In particular, for the $\mathrm{P} / \mathrm{C} / \mathrm{A}$ and $\mathrm{P} / \mathrm{FC} / \mathrm{A}$ scaffold, the color density was much higher compared to the $\mathrm{P}$ and $\mathrm{C} / \mathrm{A}$ scaffolds, meaning increased calcium mineralization. 
(a)

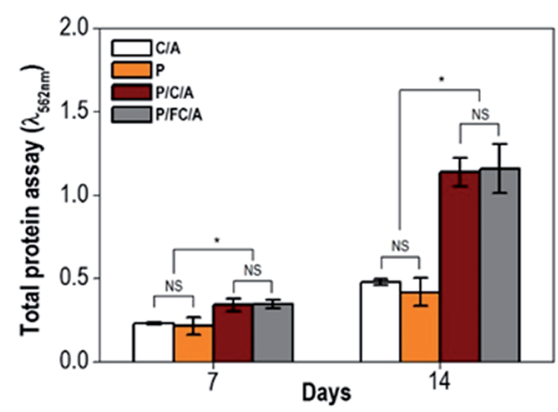

(c)

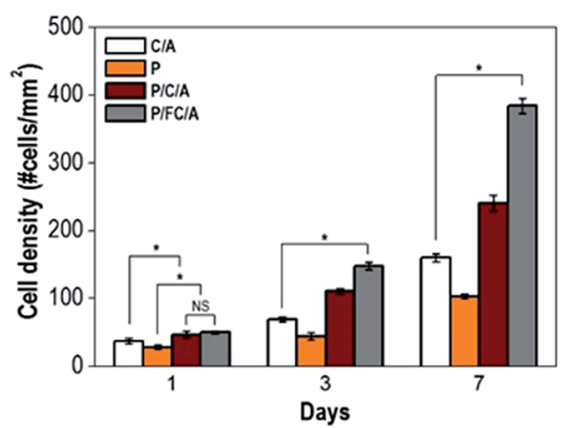

(b)
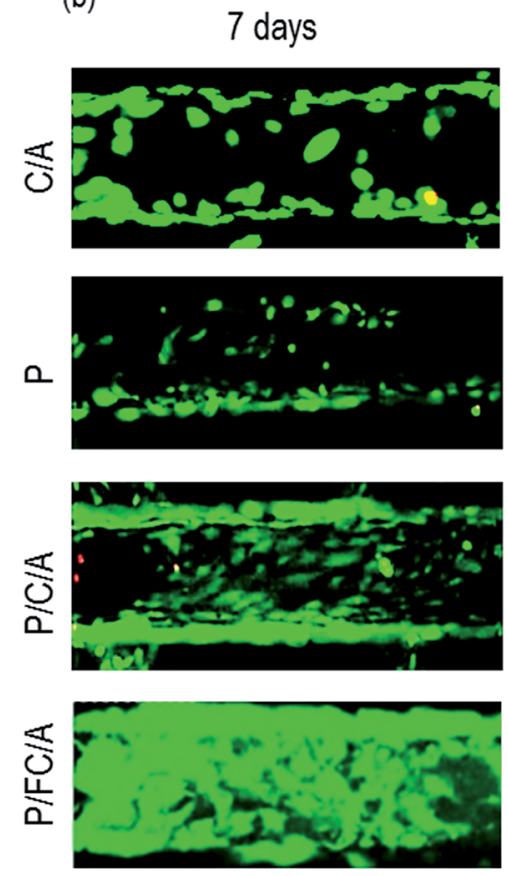

(d)

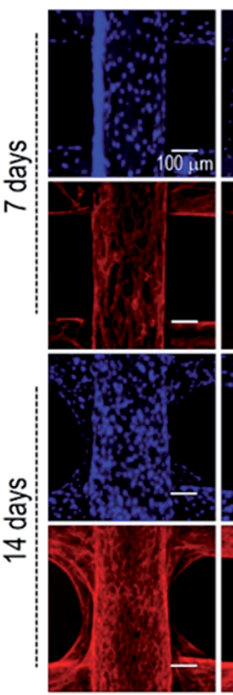

$P$
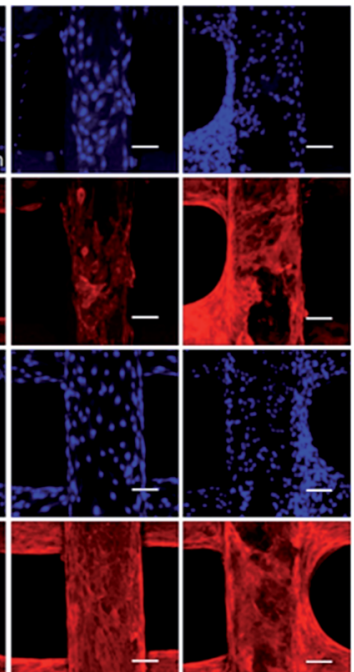

$\mathrm{P} / \mathrm{C} / \mathrm{A}$
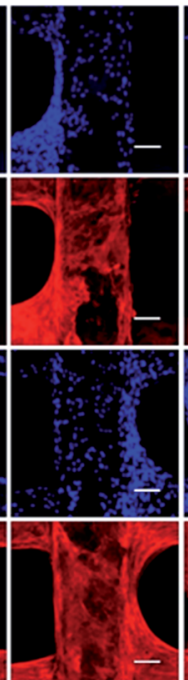

PI/FC/A

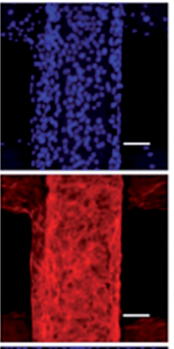

(e)
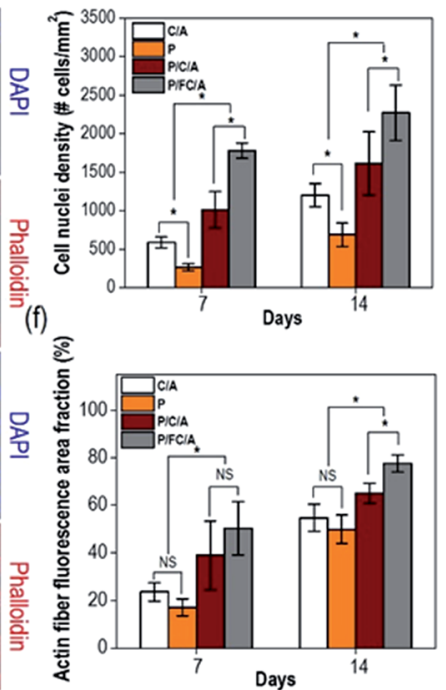

Fig. 7 (a) Total protein absorption of the biocomposites after 7 and 14 days of cell culture. (b) Live/dead images after cell culture for 7 days. (c) Cell density after culture periods of 1, 3, and 7 days. (d) DAPI (blue) and phalloidin (red) images after culture for 7 and 14 days on the biocomposites. (e) Number of cells and (f) area fraction of F-actin on the composite scaffolds. * indicates $p<0.05$ and NS indicates nonsignificance.

The relative optical density (OD) of the red-stained scaffolds, normalized to total protein content, is displayed in Fig. 8(b). The P/FC/A scaffold showed a significantly higher calcium deposition of about 1.4-fold that of the control (C/A scaffold). The result can be attributed to the combined effects of the bioactive component, phlorotannin, and the fish collagen/ alginate components promoting osteogenic differentiation of the cells. However, as shown in the result, there is no significant difference of relative calcium deposition between P/FC/A and P/ C/A scaffolds.

\subsection{In vivo tests}

In the in vivo study, we used the scaffolds (C/A and $\mathrm{P} / \mathrm{FC} / \mathrm{A})$ to observe osteogenesis in the muscle pocket in mice (Fig. 9(a)). The gross appearance showed round-shaped ectopic bone formation in both groups and histological sections showed new bone formation, with the exception of the pores, that are the spaces between the PCL struts. Osteoconduction of the scaffolds was evident in both groups (Fig. 9(b and c)). The control group showed a limited blood vessel network indicated by arrow within the osseous constructs while there were many blood vessels (red 


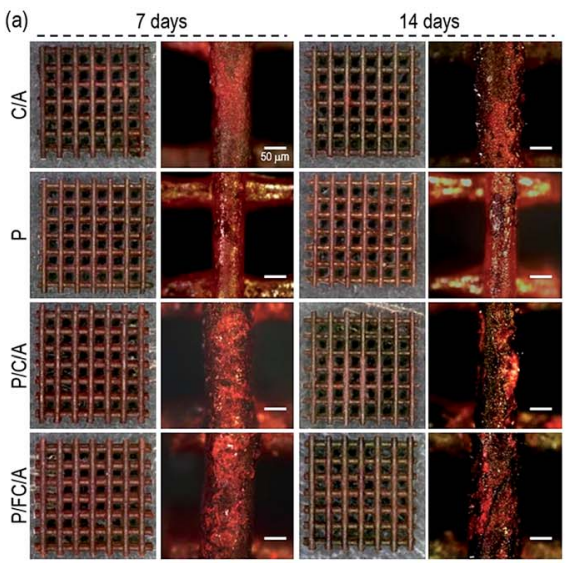

(b)

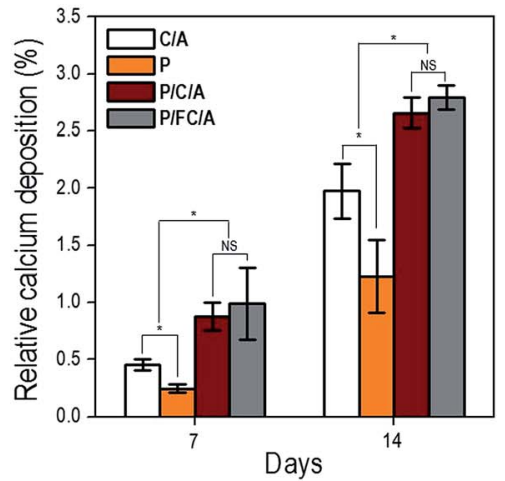

Fig. 8 (a) Optical images of alizarin red S (ARS) staining of the scaffolds after 7 and 14 days in culture. (b) Relative levels of calcium deposition on biocomposites at 7 and 14 days. * indicates $p<0.05$.
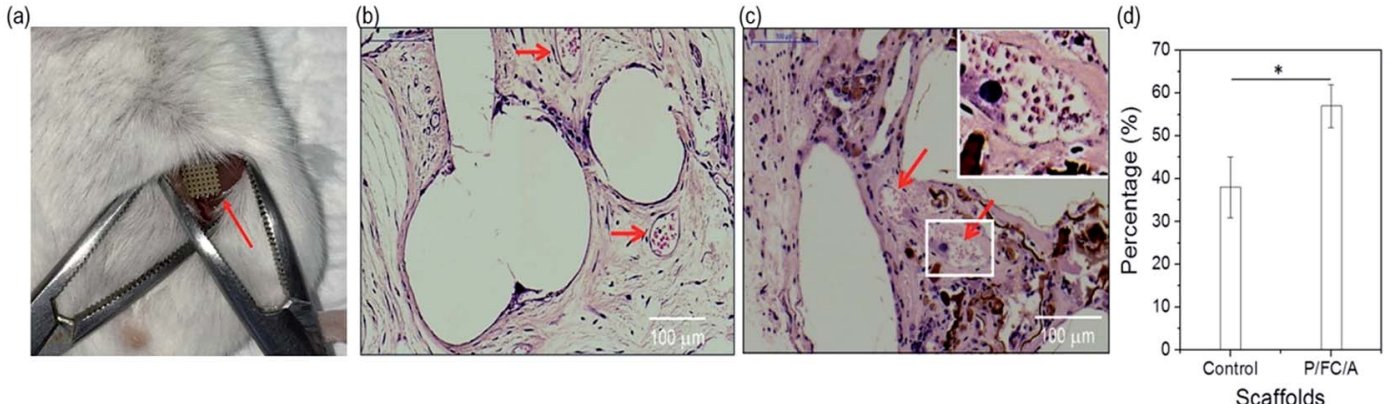

Fig. 9 (a) Subcutaneous implantation of the scaffold (P/FC/A). Histological section showing new bone formation with (b) control (C/A) and (c) phlorotannin/fish collagen/alginate (P/FC/A) scaffolds. The red arrows indicate blood vessels. (d) Histomorphometric analysis. * indicates $p<$ 0.05 .

arrows) found within the $\mathrm{P} / \mathrm{FC} / \mathrm{A}$ constructs. Histomorphometric analysis showed significantly enhanced osteogenesis with the $\mathrm{P} /$ FC/A scaffold compared to the control (Fig. 9(d)).

\section{Conclusion}

In this study, we evaluated the feasibility of fish collagen as a bioactive component to regenerate bone tissue. By employing a combinatorial process of melt-printing/simple coating, the biocomposites consisting of fish collagen/alginate and phlorotannin were fabricated. The construction of biocomposites was a layer-by-layer structure with homogeneous pore size and strut size. The coating agents on the scaffolds provided a good hydrophilic surface and induced meaningful wetting ability. The $\mathrm{P} / \mathrm{FC} / \mathrm{A}$ scaffold showed good in vitro biomineralization in SBF solution compared to the control due to the relatively good solubility of fish collagen. Although the P/C/A using the porcine collagen showed highly efficient to in vitro cellular activities, the newly designed biocomposite (P/FC/A) also showed significantly high cell proliferation and calcium deposition compared to the control (C/A). Through various in vitro and in vivo cellular activities, we have proved that the biocomposite including fish collagen supplemented with phlorotannin can be a potentially significant biomaterial for enhancing bone formation.

\section{Acknowledgements}

This study was supported by a grant from the National Research Foundation of Korea grant funded by the Ministry of Education, Science, and Technology (MEST) (Grant no. NRF2015R1A2A1A15055305), and also supported by grant from Marine Technology Application Program (PJT200673).

\section{References}

1 A. Khademhosseini, R. Langer, J. Borenstein and J. P. Vacanti, Proc. Natl. Acad. Sci. U. S. A., 2006, 103, 24802487.

2 S. F. Yang, K. F. Leong, Z. H. Du and C. K. Chua, Tissue Eng., 2001, 7, 679-689.

3 H. J. Sung, C. Meredith, C. Johnson and Z. S. Galis, Biomaterials, 2004, 25, 5735-5742.

4 D. W. Hutmacher, T. Schantz, I. Zein, K. W. Ng, S. H. Teoh and K. C. Tan, J. Biomed. Mater. Res., 2001, 55, 203-216.

5 S. Browne, D. I. Zeugolis and A. Pandit, Tissue Eng., Part A, 2013, 19, 1491-1494.

6 H. Lee, M. Yeo, S. Ahn, D. O. Kang, C. H. Jang, H. Lee, G. M. Park and G. H. Kim, J. Biomed. Mater. Res., Part B, 2011, 97, 263-270. 
7 P. Angele, J. Abke, R. Kujat, H. Faltermeier, D. Schumann, M. Nerlich, B. Kinner, C. Englert, Z. Ruszczak, R. Mehrl and R. Mueller, Biomaterials, 2004, 25, 2831-2841.

8 G. A. Edwards and G. Roberts, Clin. Mater., 1992, 9, 211-223.

9 Y. Hiraoka, Y. Kimura, H. Ueda and Y. Tabata, Tissue Eng., 2003, 9, 1101-1112.

10 Y. Kim and G. Kim, J. Mater. Chem. B, 2013, 1, 3185-3194.

11 S. Ahn, Y. Kim, H. Lee and G. Kim, J. Mater. Chem., 2012, 22, 15901-15909.

12 H. Cao, M. M. Chen, Y. Liu, Y. Y. Liu, Y. Q. Huang, J. H. Wang, J. D. Chen and Q. Q. Zhang, Colloids Surf., B, 2014, 136, 1098-1106.

13 J. Elango, J. Y. Zhang, B. Bao, K. Palaniyandi, S. J. Wang, W. H. Wu and J. S. Robinson, Int. J. Biol. Macromol., 2016, 91, 51-59.

14 S. Yamada, K. Yamamoto, T. Ikeda, K. Yanagiguchi and Y. Hayashi, BioMed Res. Int., 2014, 2014, 302932.

15 H. J. Lee, S. H. Ahn and G. H. Kim, Chem. Mater., 2012, 24, 881-891.

16 T. A. Becker, D. R. Kipke and T. Brandon, J. Biomed. Mater. Res., 2001, 54, 76-86.

17 J. L. Drury and D. J. Mooney, Biomaterials, 2003, 24, 43374351.

18 B. Ryu, Y. Li, Z. J. Qian, M. M. Kim and S. K. Kim, Chem.-Biol. Interact., 2009, 179, 192-201.

19 P. Chandika, S. C. Ko, G. W. Oh, S. Y. Heo, V. T. Nguyen, Y. J. Jeon, B. Lee, C. H. Jang, G. Kim, W. S. Park, W. Chang, I. W. Choi and W. K. Jung, Int. J. Biol. Macromol., 2015, 81, 504-513.
20 S. J. Heo, S. C. Ko, S. H. Cha, D. H. Kang, H. S. Park, Y. U. Choi, D. Kim, W. K. Jung and Y. J. Jeon, Toxicol. in Vitro, 2009, 23, 1123-1130.

21 M. Yeo, W. K. Jung and G. Kim, J. Mater. Chem., 2012, 22, 3568-3577.

22 P. S. Eggli, W. Muller and R. K. Schenk, Clin. Orthop. Relat. Res., 1988, 232, 127-138.

23 N. J. Hallab, K. J. Bundy, K. O'Connor, R. L. Moses and J. J. Jacobs, Tissue Eng., 2001, 7, 55-71.

24 Y. Kim and G. Kim, Colloids Surf., B, 2015, 125, 181-189.

25 P. M. Gallop and S. Seifter, Methods Enzymol., 1963, 6, 635641.

26 S. Koutsopoulos, J. Biomed. Mater. Res., 2002, 62, 600-612.

27 K. Rezwan, Q. Z. Chen, J. J. Blaker and A. R. Boccaccini, Biomaterials, 2006, 27, 3413-3431.

28 C. H. Lee, A. Singla and Y. Lee, Int. J. Pharm., 2001, 221, 1-22. 29 C. H. Jang, S. H. Ahn and G. H. Kim, Int. J. Biol. Macromol., 2016, 10, 1016.

30 D. W. Hutmacher, Biomaterials, 2000, 21, 2529-2543.

31 J. Glowacki and S. Mizuno, Biopolymers, 2008, 89, 338-344.

32 H. Yoon and G. Kim, J. Pharm. Sci., 2011, 100, 424-430.

33 Y. Tabata, Tissue Eng., 2003, 9, S5-S15.

34 H. H. Sun, T. J. Qu, X. H. Zhang, Q. Yu and F. M. Chen, Biotechnol. Prog., 2012, 28, 3-20.

35 G. Jin and G. Kim, Soft Matter, 2012, 8, 6264-6272.

36 Y. Koo, H. Lee, S. Kim, N. J. Song, J. M. Ku, J. Lee, C. H. Choi, K. W. Parka and G. Kim, RSC Adv., 2015, 5, 44943-44952.

37 F. H. Heijmen, J. S. du Pant, E. Middelkoop, R. W. Kreis and M. J. Hoekstra, Biomaterials, 1997, 18, 749-754. 